\title{
Incorporación del cáncer gástrico al GES: efecto sobre el acceso y oportunidad de tratamiento quirúrgico en un hospital del SSMS
}

\author{
SEBASTIÁN AGUIRRE(1) ${ }^{(1)}$ ANDRÉS ALVO ${ }^{(1)}$ y ANDRÉS ANGULO ${ }^{(1)}$
}

\section{RESUMEN}

El cáncer gástrico es una patología de muy alta incidencia y mortalidad en Chile, razón que motivó su incorporación al programa de garantías explícitas en salud, GES, desde el $1^{\circ}$ de julio de 2006. El propósito de este estudio es evaluar el efecto de este programa sobre el acceso al diagnóstico y tratamiento oportuno de esta neoplasia, para lo cual se analizaron datos secundarios obtenidos a partir de fichas clínicas, informes de Endoscopía Digestiva Alta y Biopsias, realizadas a 76 pacientes tratados quirúrgicamente por diagnóstico de cáncer gástrico entre el $1^{\circ}$ de julio 2003 hasta el 30 de junio de 2007 en un hospital del SSMS.

Los resultados mostraron que en contra de lo esperado, no hubo cambios significativos en el número de pacientes operados. De hecho, en comparación a años anteriores el número de gastrectomías por cáncer gástrico fue menor, realizándose sólo 12 en el período Post-Auge en comparación a las 16,33 gastrectomías/año del periodo precedente, lo que representa una disminución del 26,53\%; sin haber cambios significativos en la proporción por sexo y la edad de los pacientes. En cuanto a las garantías explícitas, hubo incumplimiento de plazos en, al menos, 2 de los 12 pacientes con diagnóstico de cáncer gástrico e indicación de resección quirúrgica. Las razones por la cual los resultados del Auge no se tradujeron en mayor número de cirugías y en un cumplimiento total de los plazos garantizados, son múltiples y no necesariamente excluyentes entre sí, y debieran ser objeto de análisis en estudios posteriores.

Palabras clave: cáncer gástrico, gastrectomía, salud pública

\section{ABSTRACT}

INCORPORATION OF GASTRIC CANCER TO THE GES: IMPACT ON THE ACCESS TO AND THE OPPORTUNITY FOR SURGICAL THERAPY AT A SSMS HOSPITAL

Gastric cancer is a pathology with a high prevalence and mortality rate in Chile. In view of the latter, such condition was incorporated to the explicit health guarantees program (GES) from July 1st 2006. The purpose of the present study is to assess the impact of such program on the access to a timely diagnosis and treatment for such neoplasia. This was achieved through the analysis of secondary data obtained from medical records, Upper GI Endoscopy and Biopsy reports, from 76 patients having undergone surgery for gastric cancer between July 1 st 2003 and June 30 th 2007 at a SSMS hospital.

The results showed that, against the expected, there were no significant changes in the number

(1) Alumnos de pregrado de Medicina. Facultad de Medicina. Universidad de Chile. Independencia 939. Santiago. Chile. andresalvo@hotmail.com 
of operated patients. In fact, compared to previous years, the number of gastrectomies for Gastric Cancer was smaller, with 12 procedures carried out during the Post-AUGE period, as compared to 16,33 gastrectomies/year during the previous period. This represents a decrease in 26,53\%, without significant proportional changes per patient gender and age. As for the explicit guarantees, there was non-fulfillment of the assured deadlines in at least 2 of the 12 patients with a diagnosis of gastric cancer and an indication for surgical therapy. The reasons why the AUGE outcomes did not result in a greater number of surgeries and in a total fulfillment of the guaranteed deadlines are manifold and not necessarily mutually excluding, and should be subject of analysis in further studies.

Keywords: gastric cancer, gastrectomy, public health

\section{INTRODUCCION}

El cáncer gástrico es uno de los principales cánceres a nivel mundial. De acuerdo al proyecto GLOBOCAN 2002 de la Agencia Internacional para la Investigación en Cáncer, que estima la incidencia, mortalidad y prevalencia de 26 cánceres a nivel mundial, el cáncer gástrico es de alta incidencia y mortalidad, ocupando el cuarto y segundo lugar, respectivamente ${ }^{1}$. De acuerdo a estos mismos datos, Chile es uno de los países con las mayores tasas de incidencia y mortalidad por cáncer gástrico en el mundo, con cifras similares a las observadas en Asia oriental ${ }^{2}$.

En Chile, las neoplasias son la segunda causa de mortalidad más importante, siendo responsables del 23,1\% de las defunciones. De ellas, el cáncer gástrico es la más importante, con una tasa de 19 por cada 100.000 habitantes. De hecho, el 2004 las neoplasias malignas del estómago causaron por si solas un 3,5\% de las muertes $^{3}$.

Actualmente, la base del tratamiento curativo contra el cáncer gástrico es la resección quirúrgica del tumor ${ }^{4}$. En este sentido, el pronóstico está relacionado directamente con la realización de un diagnóstico oportuno y de una correcta estadificación, pues el cáncer gástrico incipiente tratado quirúrgicamente tiene una sobrevida a 5 años entre $85-95 \%$, mientras que la sobrevida del cáncer avanzado es de sólo un $20 \%$ en el mismo período 5 .

Debido a la alta incidencia y mortalidad de esta neoplasia y a la importancia de un diagnóstico rápido y tratamiento oportuno, el cáncer gástrico ha sido incluido desde el $1^{\circ}$ de julio de
2006 como una de las patologías con garantías explícitas en el plan Auge (Acceso Universal con Garantías Explícitas), estableciéndose plazos específicos para las distintas intervenciones del equipo de salud, entre ellos un plazo no mayor a 30 días desde la confirmación diagnóstica hasta el tratamiento quirúrgico ${ }^{6}$.

El plan Auge es un programa basado en garantizar el acceso y oportunidad de diagnóstico, tratamiento y cobertura financiera para patologías con alto impacto en la salud pública nacional por su alta incidencia, mortalidad y años de vida perdidos, y que poseen un tratamiento efectivo o prevención eficaz. El plan ha sido aplicado progresivamente a partir del 2002, sumando cada año nuevas patologías con Garantías Explícitas en Salud (GES) desde el 2005.

A pesar de que el objetivo es garantizar el acceso y tratamiento oportunos, se ha reportado que para una de las patologías GES más antiguas, la insuficiencia renal crónica, las condiciones posteriores a la implementación del Auge empeoraron en términos de listas de espera más largas y deterioro de la calidad de la atención ${ }^{7-8}$.

Sin embargo, y a pesar de estos antecedentes, los estudios que evalúen el impacto del GES sobre las patologías garantizadas son escasos.

Más que el análisis detallado del funcionamiento de este Plan, el objetivo del trabajo es evaluar el efecto que tiene la implementación de un programa de salud pública -GES- sobre una patología determinada -cáncer gástrico-, describir cambios en las características de los pacientes -sexo y edad-, acceso oportuno a diagnóstico y tratamiento -endoscopía digestiva alta y cirugía- y los plazos entre ambos. 
Figura 1. Distribución mundial de la incidencia de cáncer gástrico, estandarizada por edad
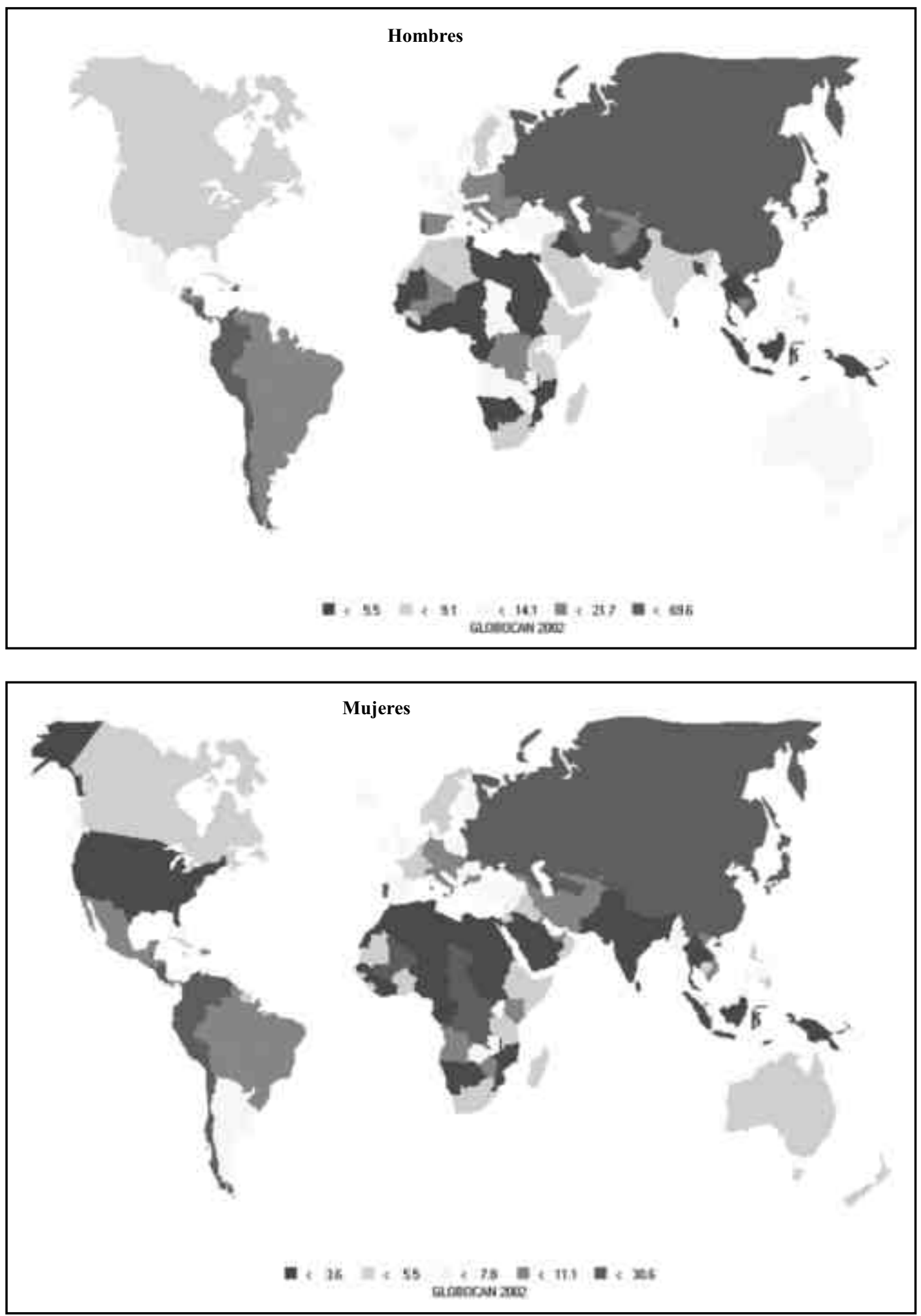
Figura 2. Número de pacientes, cirugías y gastrectomías en períodos pre y post-Auge

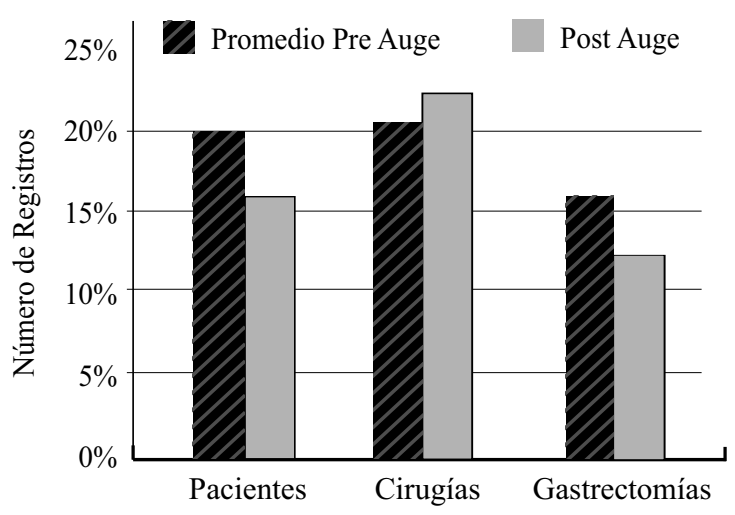

\section{MATERIAL Y MÉTODO}

Este es un estudio observacional analítico histórico prospectivo, en base a datos secundarios obtenidos a partir de fichas clínicas, informes de Endoscopía Digestiva Alta (EDA) y biopsias.

Se incluyeron todos los pacientes operados de cáncer gástrico en un hospital del SSMS entre el $1^{\circ}$ julio de 2003 al 30 de junio de 2007 $(n=76)$. Las cirugías se dividieron en dos grupos,

Figura 3. Tipo de cirugías realizadas en períodos pre y post Auge

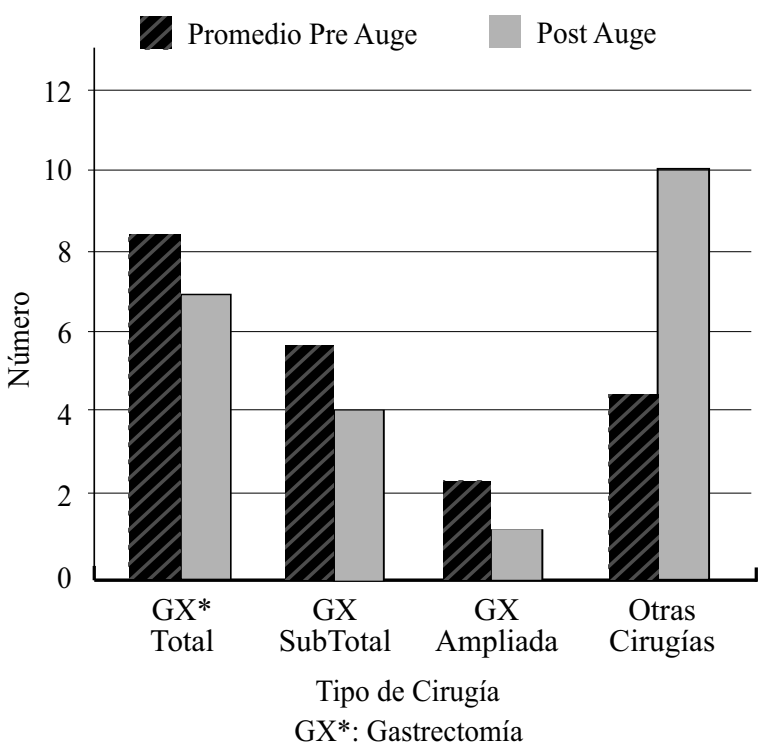

gastrectomías y otros (yeyunostomía y laparotomía exploradora). Las siguientes variables fueron estudiadas para el subgrupo de pacientes gastrectomizados $(n=61)$ : sexo, edad al momento de la cirugía, fecha de la EDA preoperatoria, tipo de gastrectomía, fecha de la cirugía y plazo EDA-Cirugía.

Los pacientes fueron divididos en dos grupos de acuerdo a la fecha en que se realizó la cirugía, "Pre-Auge" entre el $1^{\circ}$ de julio de 2003 y el 30 de junio de 2006 y "Post-Auge" entre el $1^{\circ}$ de julio de 2006 y el 30 de junio de 2007.

Los datos fueron registrados y tabulados con el software Microsoft ExcelTM. Se utilizó el

Figura 4. Distribución por sexo de los pacientes gastrectomizados en Períodos Pre $(n=49)$ y Post Auge $(n=12)$
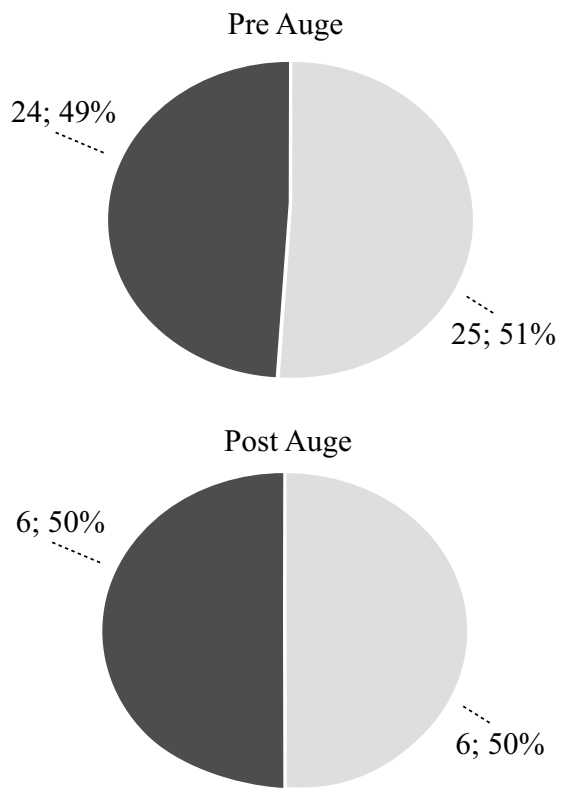

Mujeres

test de promedios con t de Student para el análisis estadístico de las variables cuantitativas y Chi cuadrado para las cualitativas, con un nivel de confianza de $95 \%$; por lo que los valores de $\mathrm{p}<0,05$ fueron considerados significativos. 


\section{RESULTADOS}

76 pacientes con diagnóstico confirmado de cáncer gástrico fueron operados entre el $1^{\circ}$ de julio de 2003 y el 30 de junio de 2007 (Figura 1), de éstos, 60 pertenecían al grupo pre-Auge (promedio por año=20) y 16 al postAuge. El porcentaje de variación post-pre Auge fue de $-20 \%$ ( -4 pacientes por año, $p>0,05)$.
Se realizaron 84 cirugías en estos pacientes (promedio por paciente $=1,11$ cirugías), 62 preAUGE (promedio por año=20,67) y 22 postAUGE. La diferencia post-pre AUGE fue de 1,33 cirugías/año $(p>0,05)$, correspondiente a un cambio de $6,45 \%$. Del total de cirugías, 61 correspondieron a algún tipo de gastrectomía $(72,62 \%)$ y 23 a otro tipo (27,38\%); en el grupo pre-Auge estos valores fueron $49(79,03 \%$;

Figura 5. Distribución por edad del total de pacientes gastrectomizados $(n=61)$

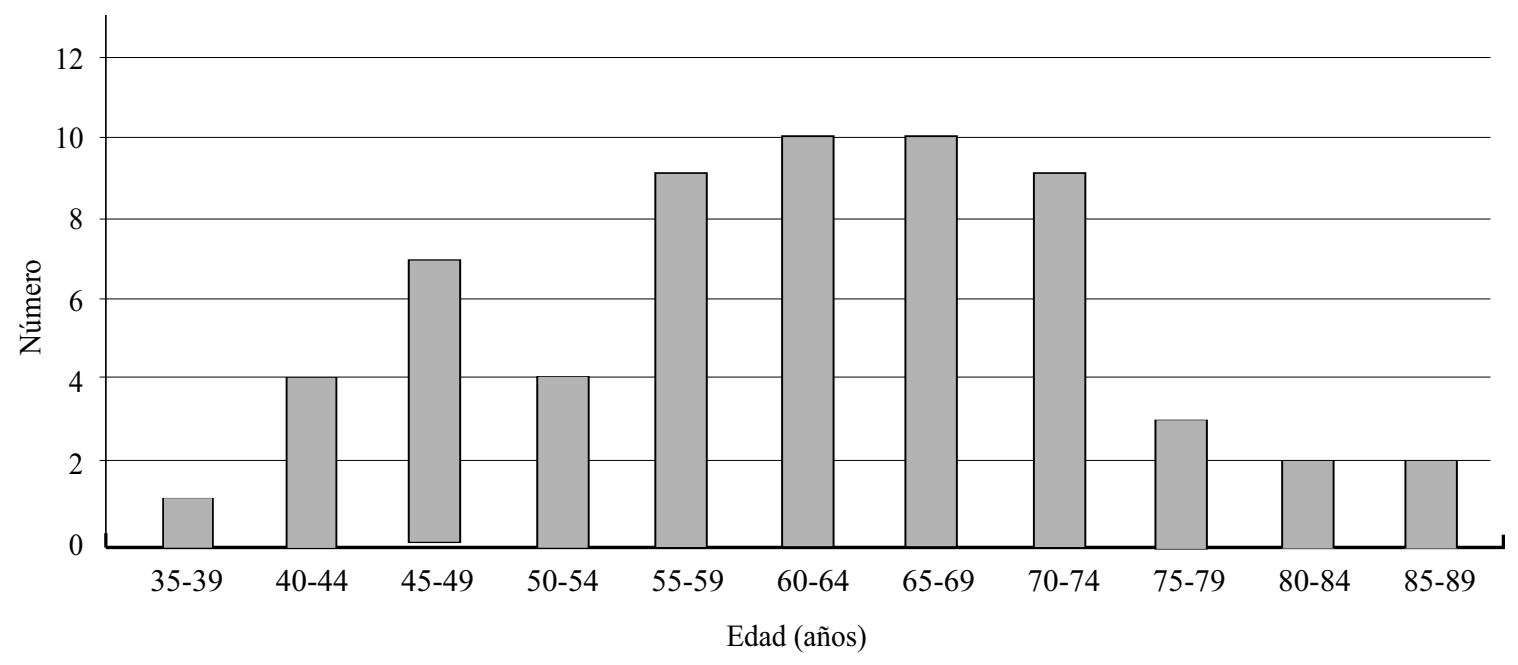

Tabla 1. Medidas de resumen de los plazos EDA-Gastrectomía Pre y Post Auge*

\begin{tabular}{|c|c|c|c|c|}
\hline & Pre-Auge & $\begin{array}{c}\text { Variación Post } \\
\text { Post-Auge }\end{array}$ & Pre-Auge & $\%$ Cambio \\
\hline Mediana & 29 & 30 & 1 & 3,45 \\
\hline IQR & 58 & 29,5 & $-28,5$ & $-49,14$ \\
\hline Promedio & 50,97 & 43,09 & $-7,88$ & $-15,46$ \\
\hline SD & 52,18 & 27,54 & $-24,64$ & $-47,22$ \\
\hline Rango & 6-204 & $9-91$ & & \\
\hline No Gastrectomías/año & 16,33 & 12 & $-4,33$ & $-26,53$ \\
\hline
\end{tabular}

*Excluyendo los pacientes con datos insuficientes para el análisis 
Figura 6. Intervalos EDA-Gastrectomía en períodos Pre $(n=49)$ y Post-AUGE $(n=12)$

Pre Auge $(n=49)$

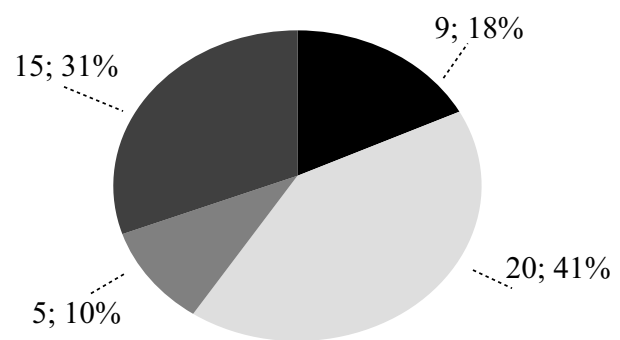

Pre Auge $(n=12)$

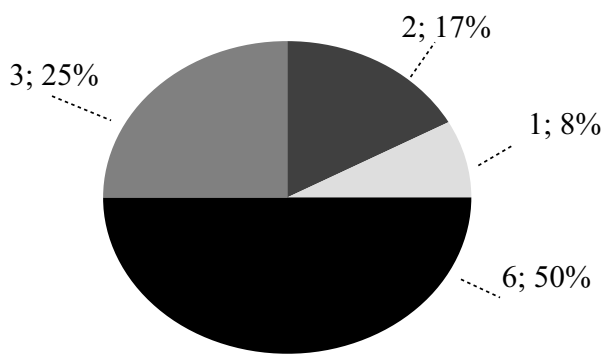

Información Insuficiente

$\leq 30$ días

31 - 60 días

$>60$ días

promedio por año=16,33) y $13(20,97 \%$; promedio por año $=4,33)$ y en el post-Auge 12 $(54,55 \%)$ y $10(45,45 \%)$, respectivamente. La variación del número de gastrectomías postpre Auge fue de $-26,53 \%$ (-4,33 pacientes/año, $\mathrm{p}>0,05)$.

Del subgrupo de pacientes gastrectomizados $(\mathrm{n}=61)$, a $32(52,46 \%)$ se les practicó una gastrectomía total, a $21(34,43 \%)$ una gastrectomía parcial y a $8(13,11 \%)$ una gastrectomía ampliada (Figura 2). Para los pacientes gastrectomizados pre-AUGE $(\mathrm{n}=49)$ estos valores fueron 25 $(51 \%), 17(34,7 \%)$ y $7(14,3 \%)$ y, para los postAuge, $7(58,33 \%), 4(33,33 \%)$ y $1(8,33 \%)$.

De acuerdo al sexo, en los pacientes gastrectomizados hubo 31 hombres y 30 mujeres, con una razón hombre:mujer (H:M) de 1,03. En el grupo pre-Auge, 25 eran hombres y 24 mujeres ( $\mathrm{H}: \mathrm{M}=1,04)$ y en el post-Auge hubo 6 hombres y 6 mujeres ( $\mathrm{H}: \mathrm{M}=1)$ (Figura 3). El cambio de la proporción entre hombres y mujeres pre y post Auge tuvo un $\mathrm{p}>0,05$.

La distribución de los pacientes según la edad al momento de la gastrectomía se muestra en la Figura 4. En promedio, la edad al momento de la gastrectomía fue de 61,54 años, con una desviación estándar (SD) de 11,79; la mediana fue de 63 años y el recorrido intercuartílico (IQR) fue 17. En los pacientes de sexo masculino, el promedio fue de 60,81 años $(S D=10,88)$ y en las de sexo femenino 62,3 años $(\mathrm{SD}=12,8)$. El promedio de edad en los pacientes gastrectomizados pre-Auge fue 61,35 años $(\mathrm{SD}=11,71)$ y post-Auge de 62,33 años $(\mathrm{SD}=12,62)$. El cambio del promedio de edad pre y post-Auge tuvo un $\mathrm{p}>0,05$.

El plazo EDA-Gastrectomía en los pacientes gastrectomizados pre-Auge fue igual o menor a 30 días en 15 pacientes $(30,61 \%)$, entre 31 y 60 días en $5(10,2 \%)$, y sobre 60 días en 9 $(18,37 \%)$. En 20 pacientes la información fue insuficiente $(40,82 \%)$. En los gastrectomizados post-Auge, los pacientes con plazos iguales o menores a 30 días fueron $6(50 \%), 3$ pacientes (25\%) estuvieron entre 31 y 60 días y $2(16,67 \%)$ superaron los 60 días; en 1 paciente $(8,33 \%)$ la información fue insuficiente (Figura 5). Las medidas de resumen para estos plazos, excluyendo los pacientes con datos insuficientes, se encuentran en la Tabla 1.

\section{DISCUSIÓN}

El plan Auge establece que los pacientes de 15 años y más con un cáncer gástrico operable tendrán garantizada su resección quirúrgica dentro de plazos bien establecidos (Figura 6), y debido a la alta frecuencia de esta patología en la población chilena, podría suponerse que al ingresar como patología GES, el número de pacientes gastrectomizados debido a cáncer gástrico aumentaría. Sin embargo, nuestros resultados muestran que ninguna de las diferencias observadas fueron significativas e incluso 
Figura 7. Plazos Auge para cáncer gástrico desde la sospecha diagnóstica hasta el tratamiento quirúrgico

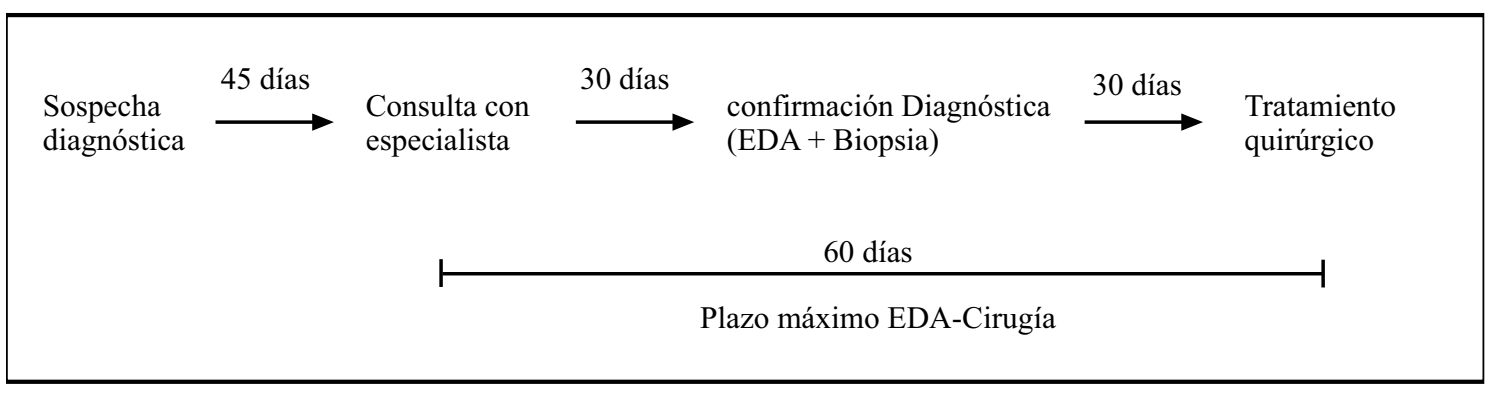

en algunas ocasiones cambiaron en el sentido opuesto a lo esperado.

Es así como el número de pacientes operados anualmente por cáncer gástrico disminuyó en 4 pacientes/año cuando se incorporó esta neoplasia al GES, esto es un $20 \%$ menos que el promedio de los 3 años anteriores. En el número de cirugías realizadas, se observó un aumento cercano al $6,5 \%$ en el periodo post-Auge, pero esto fue a costa de cirugías no curativas, sino utilizadas como diagnóstico, para tratamiento de complicaciones o con fin paliativo. Por otro lado, el número de gastrectomías/año disminuyó desde 16,33 a 12 después del $1^{\circ}$ de julio del 2006 , una disminución de $26,5 \%$ respecto del periodo pre-Auge.

El por qué de esta disminución (no significativa) en el número de pacientes operados podría corresponder a diversas causas. En primer lugar, podría deberse a que como el cáncer gástrico se convertiría en una patología GES, más hospitales se prepararon para tratarlo, por lo que aumentó la dispersión de estos pacientes en los establecimientos del sistema público de salud. También podría deberse a una política más rígida respecto a la población de pacientes operados en este hospital, suponiendo que antes del Auge, pacientes provenientes de zonas no correspondientes al hospital eran operados en él. Otra posible alternativa es que esto corresponda a problemas del proceso diagnóstico del cáncer gástrico generado por el Auge, por ejemplo, que las listas de espera para las consultas o EDA estén alargándose por incapacidad del sistema de adaptarse a la mayor demanda. Finalmente, podría deberse a problemas intrínsecos del hospital, como una disminución en el número total de cirugías realizadas ese año.

El análisis de lo ocurrido con el plazo entre la EDA y la gastrectomía, podría orientar respecto a cuál es la verdadera causa de la disminución de pacientes operados de cáncer gástrico, pero desgraciadamente el alto número de datos insuficientes hizo que esta información fuese inválida desde el punto de vista estadístico, lo que restringe de manera importante cualquier conclusión respecto a este punto. A pesar de lo anterior, sí es posible realizar dos alcances a partir de estos datos: en, al menos, 2 de los 12 pacientes (16,67\%), los plazos garantizados por el Auge no se cumplieron (Figura 6); el pequeño número de pacientes y la ausencia de comparación válida con el período anterior impiden saber si este es un fenómeno en corrección. Debe considerarse que la razón de este incumplimiento puede corresponder también a imposibilidad de operar al paciente en la fecha propuesta, debido a que no cumple los requerimientos mínimos necesarios para una cirugía. Por otra parte, si sólo se consideran los datos disponibles, en el período post-Auge, el número de días promedio entre EDA y gastrectomía disminuyó desde 51 a 43.

Además debe destacarse que la implementación del GES tuvo un impacto positivo en la disponibilidad de datos, ya que el número de datos insuficientes se redujo de $41 \%$ a $8 \%$.

En cuanto a la edad de los pacientes, no hubo cambios entre los períodos pre y post-Auge, lo que puede sugerir que el Auge no tuvo un efecto importante sobre el diagnóstico precoz de la enfermedad, pues los pacientes se están operando 
a la misma edad. Por este motivo, sería importante evaluar qué es lo que está ocurriendo a nivel de sospecha diagnóstica y si es que los pacientes con síntomas de cáncer gástrico están logrando acceder a consultar oportunamente, considerando que el cáncer gástrico avanzado es letal sin cirugía, con una sobrevida cercana a los seis meses ${ }^{9}$. Además, debido a la alta incidencia y mortalidad del cáncer gástrico en nuestro país, es necesario evaluar la posibilidad de incluir la realización de pruebas de tamizaje en los grupos de mayor riesgo, y si su costoefectividad lo justifica. En este sentido, existen estudios a nivel internacional y nacional que así lo avalan ${ }^{10-13}$.

Respecto a la distribución por sexo, se observó que la relación hombre:mujer no varió entre ambos periodos y que era muy cercana a 1. Este es un hallazgo interesante, puesto que se sabe que el cáncer gástrico afecta predominantemente a los hombres ${ }^{14-16}$. Si esta situación se debe a que las mujeres están siendo diagnosticadas en estadios más iniciales o consultando más precozmente, si se debe a características propias de la población atendida por este hospital o si presentan un mayor porcentaje de casos operables por condiciones biológicas inherentes al sexo, debe ser evaluado.

\section{CONCLUSIÓN}

A partir de la incorporación del cáncer gástrico dentro de las patologías GES, el número de pacientes gastrectomizados por cáncer gástrico en este hospital no cambió significativamente, e incluso disminuyó. Asimismo, no hubo cambios en la edad de los pacientes al momento de la cirugía. En al menos 2 de los 12 pacientes operados dentro del GES, los plazos establecidos no se cumplieron. Por otra parte, el número promedio de días entre la EDA y la gastrectomía disminuyó con el GES, así como la variabilidad de este plazo, respecto al período pre-Auge.

El motivo de estos resultados aparentemente opuestos a lo esperado con el plan Auge puede deberse a múltiples causas y deben realizarse estudios adicionales para aclarar la verdadera explicación. La reciente incorporación de la patología estudiada al GES no permite establecer si éste es un fenómeno en corrección, transitorio o estable. A nuestro juicio esto no deja de hacer relevante a este trabajo en términos de describir la relación entre la implementación de esta medida de salud pública y los efectos sobre la entidad prestadora del servicio garantizado. Finalmente, debe considerarse que estos resultados reflejan la situación ocurrida en un solo establecimiento, por lo que su reproducibilidad en el contexto regional y nacional debe ser, asimismo, evaluada.

\section{AGRADECIMIENTOS}

Agradecemos a las siguientes personas por su importante ayuda en la realización de este trabajo: Paula Palma Albornoz, Ingrid Osorio González, Genaro Villalobos López, Pedro Arrate Miranda, y a los Dres. Rainier Aguirre Sánchez y Julia González.

\section{REFERENCIAS}

1. PARKIN DM, BRAY F, FERLAY J, PISANI P. Global cancer statistics, 2002. CA Cancer J Clin. 2005 MarApr;55(2):74-108.

2. FERLAY J, BRAY F, PISANI P, PARKIN DM. GLOBOCAN 2002. Cancer Incidence, Mortality and Prevalence Worldwide. IARC Cancer Base No. 5 Version 2.0. Lyon, France: IARCPress; 2004.

3. MINISTERIO DE SALUD, Departamento de Estadísticas e Información de Salud. Estadísticas vitales. Disponible en:

http://deis.minsal.cl/deis/indexmc.asp [Consultado el 25 de Agosto de 2007]

4. MOEHLER M, GALLE PR, GOCKEL I, JUNGINGER T, SCHMIDBERGER H. The multidisciplinary management of gastrointestinal cancer. Multimodal treatment of gastric cancer. Best Pract Res Clin Gastroenterol. 2007;21(6):965-81

5. CSENDES P, SANHUEZA A. Evaluación del Cáncer gástrico por tomografía computada. Rev. Chil. Radiol., 2006, vol.12, no.2, p.57-63.

6. MINISTERIO DE SALUD. Segundo régimen de garantías explícitas en salud, 2006. Disponible en: http://www.supersalud.cl/normativa/571/articles837 recurso_1.pdf [Consultado el 25 de Agosto de 2007]

7. GONZÁLEZ F. Implementación del plan AUGE en pacientes con IRC. Rev. méd. Chile, mayo 2003, vol.131, no.5, p.545-551.

8. GONZÁLEZ F. La implementación del Plan de Acceso Universal y Garantías Explícitas (Plan AUGE) ha 
deteriorado la calidad del tratamiento de pacientes con insuficiencia renal terminal. Rev. méd. Chile, oct. 2006, vol.134, no.10, p.1288-1294.

9. TOSHIFUSA NAKAJIMA. Gastric cancer treatment guidelines in Japan. Gastric Cancer, 2002, 5: 1-5.

10. TASHIRO A, SANO M, KINAMERI K, FUJITA K, TAKEUCHI Y. Comparing mass screening techniques for gastric cancer in Japan. World J Gastroenterol. 2006 Aug 14;12(30):4873-4.

11. DAN YY, SO JB, YEOH KG. Endoscopic screening for gastric cancer. Clin Gastroenterol Hepatol. 2006 Jun;4(6):709-16.

12. CALVO BELMAR A, PRUYAS M, NILSEN E, VERDUGO P. Populational research of gastric cancer in digestive symptomatic patients, from 1996 to 2000. Rev Med Chil. 2001 Jul;129(7):749-55.

13. LLORENS P. Gastric cancer mass survey in Chile and its value in a symptomatic group. No. 485, Abstract Book - The World Congresses in Stockholm Sweden. June 1982; 14-19
14. Yamamoto S. Stomach cancer incidence in the world. Jpn J Clin Oncol. 2001 Sep;31(9):471

15. AHN YO, PARK BJ, YOO KY, KIM NK, HEO DS, LEE JK, AHN HS, KANG DH, KIM H, LEE MS, et al. Incidence estimation of stomach cancer among Koreans. J Korean Med Sci. 1991 Mar;6(1):7-14.

16. MEDINA E, KAEMPFFER A. Mortalidad por cáncer en Chile: consideraciones epidemiológicas. Rev. méd. Chile, oct. 2001, vol.129, no.10, p.1195-1202.

Recepción: 11 diciembre 2007 Aprobación: 28 marzo 2008

Usted puede comentar éste y otros artículos publicados en la Revista Chilena de Salud Pública, enviando un correo electrónico a revistasp@med.uchile.cl 